Note

\title{
Repression of Acute Gastric Mucosal Lesions by Antioxidant-Containing Fraction from Fermented Products of Okara (Bean-Curd Residue)
}

\author{
Takashi YoKota, ${ }^{*}$ Hiroshi OHAmi, Hihumi OHISHI, ${ }^{1}$ \\ Takashi HATTORI, ${ }^{1}$ and Kenji WATANABE ${ }^{2}$ \\ Division of Pathology, Institute of Gerontology, Nippon Medical School, \\ Kawasaki 211, Japan \\ ${ }^{1}$ Kyodo Milk Industry Co., Ltd., Nishitama-gun, Tokyo 190-01, Japan \\ ${ }^{2}$ The United Graduate School of Agriculture Science, \\ Gifu University, Gifu 501-11, Japan \\ (Received March 31, 1995)
}

\begin{abstract}
Summary A crude antioxidant preparation from fermented okara (NTX) was examined for its protection against the pathogenesis of gastric ulcer in water-immersed rats. The areas of gastric mucosal lesions as well as the levels of thiobarbituric acid-reactive substances, prostaglandin $\mathrm{E}_{2}$ and hyaluronic acid in the gastric mucosa were measured in relation to the time elapsed after the imposition of stress. Comparison with those parameters in $\alpha$-tocopherol-treated groups revealed that NTX exerted an anti-inflammatory effect on gastric injury, probably by functioning as a free radical scavenger.

Key Word anti-inflammatory activity, gastric mucosal lesions, lipid peroxidation, prostaglandin $\mathrm{E}_{2}$, hyaluronic acid, water-immersed restraint stress
\end{abstract}

Conflicting views have been expressed regarding the factors responsible for gastric mucosal lesions arising from various types of stress. Such factors include reduction of the blood flow by hemorrhagic hypotension, acceleration of gastric acid secretion, and lowering of intramucosal prostaglandin $\mathrm{E}_{2}\left(\mathrm{PGE}_{2}\right)$. In fact, however, the pathogenesis of mucosal lesions is not fully understood yet. Much attention has been recently focused on the role of active oxygen species in the form of superoxide anion, hydroxyl radical, and lipid peroxyl radical in mediating ischemic tissue damage (1). Furthermore, lipid peroxidation mediated by free radicals is believed to be one of the important causes of cell membrane destruction and cell damage, since the cell membrane contains unsaturated lipids (2). In previous papers $(3,4)$, a crude antioxidant preparation from fermented soybean

\footnotetext{
* To whom correspondence should be addressed.
} 
products (natto) was proved to exert antioxidative activities in vitro and in vivo. Such a fraction from fermented okara was also shown to exhibit an anti-inflammatory effect on rat foot pad-edema when administered orally (5).

For this reason, we studied the effect of the antioxidant-rich fraction from fermented okara (NTX) on the prevention or repair of gastric mucosal lesions in rats under water-immersion stress.

\section{Materials and methods}

Preparation of NTX. Okara was fermented in our laboratory with Bacillus natto at $45^{\circ} \mathrm{C}$ for $24 \mathrm{~h}$ in such a manner as to prepare natto. The fermented product was extracted with methanol, and then concentrated in vacuo. The $\mathrm{pH}$ of the concentrate was adjusted to 3.5 with $0.1 \mathrm{~N} \mathrm{HCl}$, and then extracted with butanol. The separated butanol phase was evaporated under reduced pressure, and the residual concentrate was extracted with ethyl ether after adjustment to $\mathrm{pH}$ 8.5. The ethyl ether layer was concentrated in vacuo, and then fractionated by HPLC to obtain the antioxidant-rich fraction 'NTX', as described in a previous paper (5).

Induction of acute gastric mucosal lesions in rats. Male rats of the $\mathrm{Jcl}$ : Wistar strain weighing about 300-350 g, which had free access to a stock diet (CE-2, Clea, Tokyo) and tap water, were used in this experiment. They were individually housed in wire cages in an air-conditioned room $\left(24 \pm 1^{\circ} \mathrm{C}\right)$ with a 12 -h light-dark cycle during the experimental period (14 days), fasted for $24 \mathrm{~h}$, and immersed in $23^{\circ} \mathrm{C}$ water for 1,3 and $6 \mathrm{~h}$ under restraint. In the first group (named NTX-OR), NTX at a constant level $(200 \mu \mathrm{g} / \mathrm{kg} /$ day $)$ had been orally administered every day for 7 days till the stress load was applied. The second group (named VE-OR) had been given $\alpha$-tocopherol $(200 \mu \mathrm{g} / \mathrm{kg} / \mathrm{day})$ under the same conditions as the first group. The third group (named NTX-IV) received an intravenous injection of 20 $\mu l$ of NTX-suspended solution $(6 \mathrm{mg} / \mathrm{ml}) 2 \mathrm{~h}$ before the stress load. The fourth group (control) had been orally given physiological saline $(1 \mathrm{ml} /$ day) under the same conditions as NTX-OR. The fifth group, in which no treatment was carried out, was designated as no-stress. The extent of gastric mucosal damage was determined by taking out the stomach immediately after sacrifice and measuring the area $\left(\mathrm{mm}^{2}\right)$ of the surface hemorrhagic erosions, and the sum of individual measurements was taken as the ulcer index.

Chemical analyses. The contents of lipid peroxide in serum and in the homogenate of stomach body were measured by the colorimetric method using thiobarbituric acid (TBA) (6). $\mathrm{PGE}_{2}$ was determined by the method of Kawano et al. (7) after part of the stomach was quickly rinsed in physiological saline containing $1 \mathrm{~mm}$ EDTA and $0.1 \mathrm{~mm}$ indomethacin at a low temperature. The gastric mucosa was treated as described by Ohishi et al. (8), and hyaluronic acid (HA) was determined by two-dimensional electrophoresis according to the method of Hata et al. (9).

Statistical analysis. The values shown represent $\mathrm{M} \pm \mathrm{SE}(n=5)$ and statistical significance was determined by Student's two-tailed test. 


\section{Results and discussion}

The total area of erosion increased with time after the load of stress by water-immersion, in the order of control, VE-OR, NTX-OR, and NTX-IV. A significant difference in the area of erosion was observed especially between the control and NTX-IV or NTX-OR groups $3 \mathrm{~h}$ after the stress load (Fig. 1). Figure 2 shows the time course of changes in TBA-reactive substances (TBARS) of gastric mucosa after the stress of water-immersed restraint. NTX was found to suppress the increase in TBARS better than VE in almost equal amounts. On the other hand, TBARS levels in the serum of all groups showed no significant changes after the water-immersion restraint stress (data not shown). Figure 3 shows the time course of changes in $\mathrm{PGE}_{2}$ levels in gastric mucosa after the stress load. The $\mathrm{PGE}_{2}$ levels increased with time in the NTX group and reached a plateau in $3 \mathrm{~h}$, while they decreased after $3 \mathrm{~h}$ in the control and VE-OR groups. Gastric mucosal HA level in the four groups tested and the no-stress one are shown in Fig. 4. The HA were down by about $40 \%$ and $26 \%$ in the control and VE groups $3 \mathrm{~h}$ after the load was applied, respectively, in comparison with those in the no-stress group. On the other hand, the HA in the NTX group was slightly higher than in the no-stress group.

Factors affecting gastric acid secretion throughout the autonomic nervous system, an increase in aggressive factors such as specific oxygens, and a decrease in defensive factors such as mucosal blood flow, have been seriously considered to underlie the pathogenesis of acute gastric mucosal lesions $(10,11)$. Recently, it has been reported that superoxide dismutase (SOD) also protects against the formation of gastric lesion induced by hemorrhagic ischemia $(12,13)$. These facts indicated

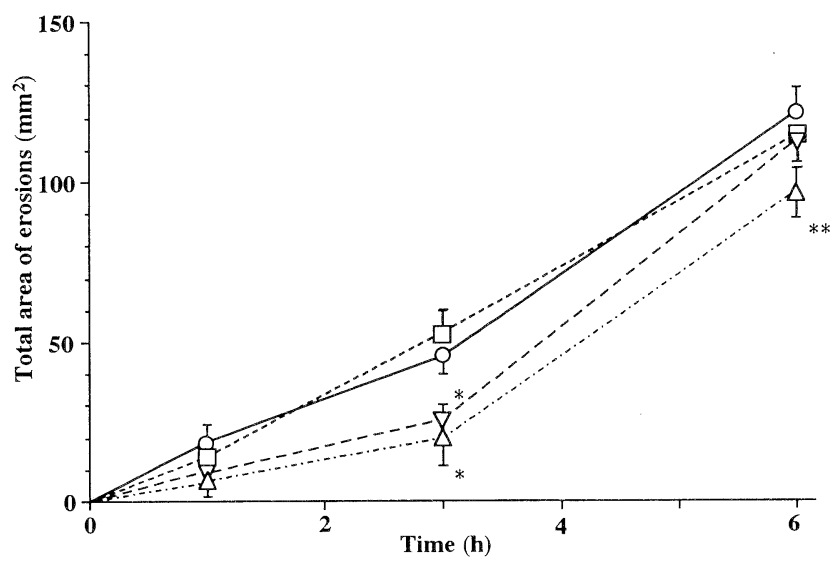

Fig. 1. Changes in erosion area in the stomach with the time elapsed after the onset of water-immersion restraint stress. Values are expressed as $\mathrm{M} \pm \mathrm{SE}$ of 5 rats. Significantly different from control at the same time after stress loading; ${ }^{*} p<$ $0.05,{ }^{*} p<0.01$. $-\bigcirc-$, control; --- $\square---$, VE-OR; -- --- , NTX-OR; $--\triangle^{--}$, NTX-IV. 


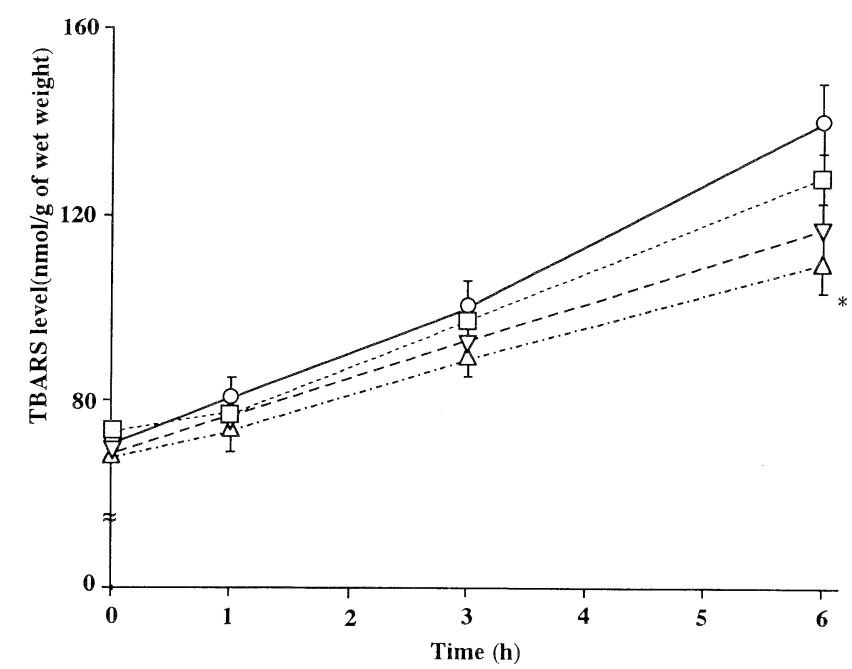

Fig. 2. Changes in TBARS levels in the gastric mucosa with the time elapsed after the onset of water-immersion restraint stress. Values are expressed as $\mathrm{M} \pm \mathrm{SE}$ of 5 rats. Significantly different from control at the same time after stress loading; ${ }^{*} p<0.01$. $-\bigcirc-$, control; --- $\square---$, VE-OR; -- $\nabla--$, NTX-OR; -- $\triangle--$, NTXIV:

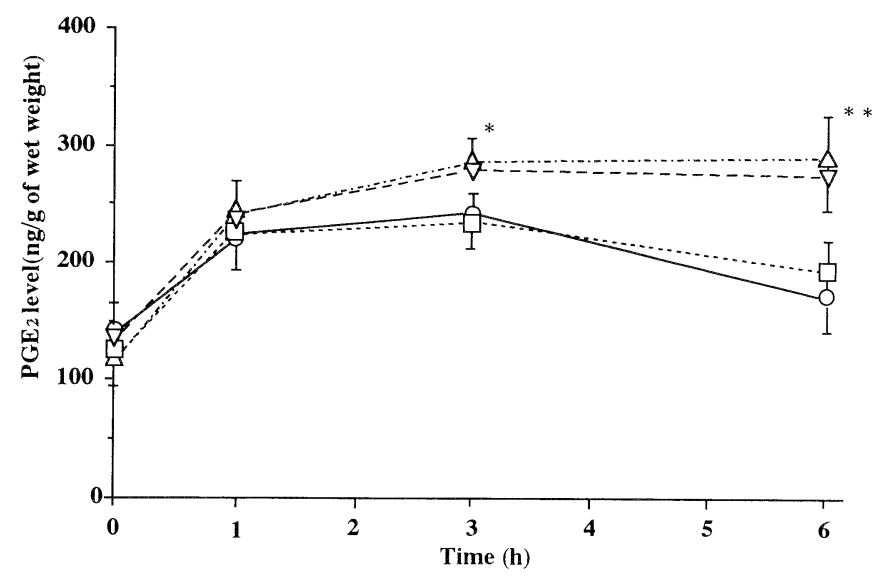

Fig. 3. Changes in $\mathrm{PGE}_{2}$ levels in the gastric mucosa with the time elapsed after the onset of water-immersion restraint stress. Values are expressed as $\mathbf{M} \pm \mathbf{S E}$ of 5 rats. Significantly different from control at the same time after stress loading; $*_{p}<0.05,{ }^{* *} p<0.01 . \quad-\bigcirc-$, control; --- $\square---$, VE-OR; -- $\nabla--$, NTX-OR; $--\triangle \cdot$, NTX-IV.

that oxygen-derived free radicals might play a role in the lesion formation, although the source of oxygen radicals in rat gastric mucosal injury has not been made clear. This study has also indicated that the propagation of peroxidation promotes tissue 


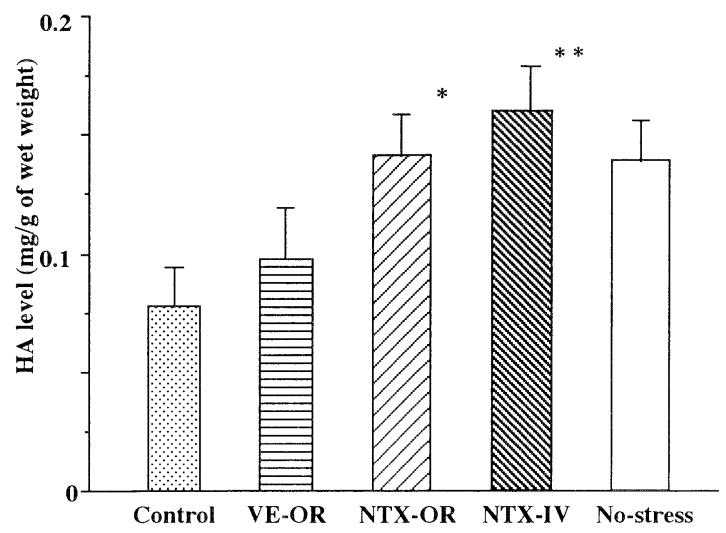

Fig. 4. Mucosal HA levels in the stomach $3 \mathrm{~h}$ after the onset of water-immersion restraint stress. The height and bar of each column represent the $\mathbf{M} \pm \mathbf{S E}$. Significantly different from control; ${ }^{*} p<0.05,{ }^{* *} p<0.01$.

damage in the gastric mucosa, and that NTX may function as an in vivo scavenger of oxygen-derived free radicals. The $\mathrm{PGE}_{2}$ level tended to decrease with the progression of mucosa erosion, as seen in the two NTX groups having the higher levels of $\mathrm{PGE}_{2}$ than the control and VE groups. Arakawa et al. (14) have described that a stress-induced decrease in intramucosal $\mathrm{PGE}_{2}$ may play an important role in the pathogenesis of ulcer formation. On the other hand, Egan et al. (15) have reported that during the generation of $\mathrm{PGH}_{2}$ from $\mathrm{PGE}_{2}$, a reductive breakdown of the product of $\mathrm{PGG}_{2}$ inactivates cyclooxygenase. Therefore, these results imply that the $\mathrm{PGE}_{2}$ synthesis has been enhanced by the presence of NTX. It has been reported that HA in the gastric mucosa is degraded by the generated superoxide anion $(16,17)$, and that its degradation is depressed by free radical scavengers such as SOD or catalase (18). The fact that the degradation of HA was inhibited by NTX administration may indicate that NTX acts as a free radical scavenger in vivo.

Both erosions and TBARS in the gastric mucosa were significantly lowered by treatment with NTX. This result also supports the protective effect of NTX against peroxidation responsible for gastric mucosal lesions. In this study, we compared the effectiveness of NTX and $\alpha$-tocopherol in vivo as antioxidants in equal amounts and showed that NTX exerted a higher anti-inflammatory effect on gastric injury than $\alpha$-tocopherol. It can not necessarily be concluded that NTX is a better antioxidant than $\alpha$-tocopherol, because the molecular weight of NTX is still obscure. Further investigation on the chemical structure and function of NTX are needed to provide insight into new therapeutic strategies.

\section{REFERENCES}

1) Yoshikawa, T., Ueda, S., Takemura, T., and Sugino, S. (1990): Role of oxygenderived free radical in the pathogenesis of gastric mucosal lesions in rats. J. Clin. 
Gastroenterol., 12, S65-S71.

2) Niki, E. (1987): Antioxidants in relation to lipid peroxidation. Chem. Phys. Lipids, 44, $227-253$.

3) Hattori, T., Ohishi, H., Yokota, T., Ohami, H., and Watanabe, K. (1995): Antioxidative effect of crude antioxidant preparation from soybean fermented by Bacillus natto. Lebensm.-Wiss. Technol., 27, 135-139.

4) Hattori, T., Ohishi, H., Yokota, T., Ohami, H., and Watanabe, K. (1995): Beneficial effect of crude antioxidant preparation from fermented soybean food on xanthine oxidase-hypoxanthine-induced foot-edema in rats. Lebensm.-Wiss. Technol., 28, 169173.

5) Yokota, T., Hattori, T., Ohishi, H., Ohami, H., and Watanabe, K. (1995): Effect of oral administration of crude antioxidant preparation from fermented products of okara (bean curd residue) on experimentally induced inflammation. Lebensm.-Wiss. Technol., in press.

6) Ohkawa, H., Ohishi, N., and Yagi, K. (1979): Assay for lipid peroxide for animal tissues by thiobarbituric acid reaction. Anal. Biochem., 95, 351-358.

7) Kawano, K., Sugita, M., Oka, M., and Tabata, N. (1989): A simple rapid and simultaneous extraction of thromboxane $B_{2}, 6$ keto-prostaglandin $F 1 \alpha$ and prostaglandin $\mathrm{E}_{2}$. Jpn. J. Inflamation, 7, 511-515.

8) Ohishi, H., Binette, J. P., and Schmid, K. (1986): Myocardial chondroitin sulfates D and $\mathrm{E}$ in a case of acute carbon monoxide poisoning. Clin. Chim. Acta, 156, 157-164.

9) Hata, R., Ohkawa, S., and Nagai, Y. (1987): Low-sulfated chondroitin sulfate in human blood and urine. Biochim. Biophys. Acta, 543, 156-162.

10) Menguy, R., and Masters, Y. E. (1974): Gastric mucosal energy metabolism and stress ulceration. Ann. Surg., 180, 538-548.

11) Manabe, T., Suzuki, T., and Honjo, I. (1977): Changes of upper gastrointestinal blood flow after hemorrhage in rabbits. Surgery, 81, 446-452.

12) Ito, M., and Guth, P. H. (1985): Role of oxygen-derived free radicals in hemorrhagic shock-induced gastric lesions in the rat. Gastroenterology, 88, 1162-1167.

13) Yoshikawa, T., Miyagawa, H., Yoshida, N., Sugino, S., and Kondou, M. (1986): Increase in lipid peroxidation in rat gastric mucosal lesions induced by waterimmersion restraint stress. J. Chin. Biochem. Nutr., 1, 271-277.

14) Arakawa, T., Kobayashi, K., Ono, T., and Yamamoto, S. (1981): Effect of waterimmersion stress on prostaglandin $\mathrm{E}_{2}$ in rat gastric mucosa. Gastroent. Jpn., 16, 236241.

15) Egan, K.W., Paxton, J., and Kuehi, F.A., Jr. (1976): Mechanism for irreversible self-deactivation of prostaglandin syntheses. J. Biol. Chem., 251, 7329-7335.

16) McCord, J. M. (1974): Free radicals and inflammation; Protection of synovial fluid by superoxide disumutase. Science, 185, 529-531.

17) Del Maestro, R. F., Thaw, H. H., Björk, J., Planker, M., and Arfors, K. E. (1980): Free radicals as mediators of tissue injury. Acta Phys. Scand. (suppl.), 492, 43-57.

18) Halliwell, B. (1978): Superoxide-dependent formation of hydroxyl radicals in the presence of iron salts. Its role in degradation of hyaluronic acid by a superoxide generating system. FEBS Lett., 96, 238-242. 